\title{
THE Q0957+561 MICROLENSING
}

\author{
DAVID J. THOMSON \\ ATET Bell Labs \\ Murray Hill, NJ 07974-2079 \\ AND \\ RUDOLPH E. SCHILD \\ Harvard-Smithsonian Center for Astrophysics \\ 60 Garden Street, Cambridge MA 02138
}

\begin{abstract}
The TwQSO microlensing shows rich structure with cusp-profiled features on time scales of weeks to months, and with periodic effects on time scales of a few years.
\end{abstract}

With the TwQSO time delay now converging to a value around 410 days (404 days, Schild \& Thomson these proceedings; 440 days, Haarsma et al. these proceedings; 423 days, Pelt et al. 1995), the microlensing on all time scales can be recognized. Some of these results have been discussed in Schild \& Thomson (1995, ST95 hereafter) to which we shall frequently refer for illustrations.

The microlensing history is illustrated as Fig 2 in ST95, together with a cubic polynomial fit. In the 15-year history of monitoring of two image components, no strong microlensing feature expected for an unresolved quasar source has been observed. Instead, we find a complex pattern of microlensing fluctuations with a characteristic time scale of $1 / 4$ year.

One of these microlensing cusps, observed in 1988, is shown as Fig 3 of ST95. Plotted is the brightness record of the A image component from 90 nights of observations. The event shows a Lorenzian profile with an amplitude of only $0.04 \mathrm{mag}$. Superimposed upon this is a pattern of positive and negative cusps with a smaller, 0.01 magnitude amplitude.

These features are entirely unexpected, and may signal the presence of a population of low-mass microlenses in lens galaxy G1. If we use a normal scaling of event duration with square root of mass, we easily determine 
a mass of $10^{-5}$ solar masses for the responsible objects. Of course other explanations may be possible.

Also unexplained at present is the structure in the Fourier power spectrum, Figure 4 of ST95. We interpret the low-frequency parts of this power spectrum as having two principal components; a continuum extending from 0 to $4 \mathrm{cycles} / \mathrm{yr}$, and several lines indicative of periodicity. The lines may indicate orbital motions of the microlensing masses, and we have found that the lines tend to be strongest in restricted parts of the data set, extending over several years. Note that due to the logarithmic scaling of this plot, the lines have a factor of 10 times the power in the adjacent continuum.

The second feature of the Fourier power spectrum is the continuum with a cutoff at 4 cycles/yr. This could signal the presence of a population of objects in the lens galaxy having planetary mass.

\section{References}

Pelt, J., et al., 1995, A\&A, in press

Schild, R. \& Thomson, D.J., 1995, Dark Matter, eds. S. Holt \& C. Bennett (New York: AIP Press) 